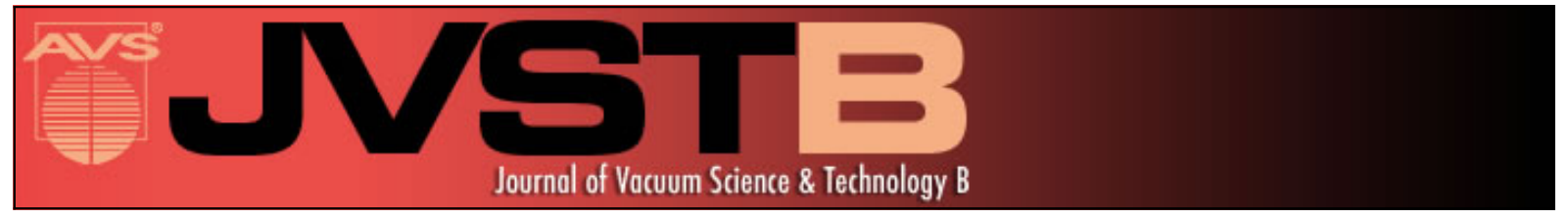

\title{
Use of spectroscopic ellipsometry for feedback control during the growth of thin AIAs layers
}

M. Beaudoin, S. R. Johnson, M. D. Boonzaayer, Y.-H. Zhang, and B. Johs

Citation: Journal of Vacuum Science \& Technology B 17, 1233 (1999); doi: 10.1116/1.590728

View online: http://dx.doi.org/10.1116/1.590728

View Table of Contents: http://scitation.aip.org/content/avs/journal/jvstb/17/3?ver=pdfcov

Published by the AVS: Science \& Technology of Materials, Interfaces, and Processing

\section{Articles you may be interested in}

Epitaxial growth of InGaAsSb/AIGaAsSb heterostructures for mid-infrared lasers based on strain engineering J. Vac. Sci. Technol. B 22, 2240 (2004); 10.1116/1.1775196

Closed-loop control of resonant tunneling diode barrier thickness using in situ spectroscopic ellipsometry J. Vac. Sci. Technol. B 18, 1439 (2000); 10.1116/1.591399

Real-time composition control of InAIAs grown on InP using spectroscopic ellipsometry J. Vac. Sci. Technol. B 18, 1435 (2000); 10.1116/1.591398

High-quality InAIAs layers grown on (411)A-oriented InP substrates by molecular beam epitaxy J. Vac. Sci. Technol. B 17, 1482 (1999); 10.1116/1.590778

In situ monitoring of $\mathrm{AIGaAs}$ compositions and $\mathrm{GaAs}$ growth at high temperature by spectroscopic ellipsometry and desorption mass spectroscopy

J. Vac. Sci. Technol. B 17, 1218 (1999); 10.1116/1.590748

\section{PIDEN}

Contact Hiden Analytical for further details: w www.HidenAnalytical.com E info@hiden.co.uk CLICK TO VIEW our product catalogue

\section{Instruments for Advanced Science}

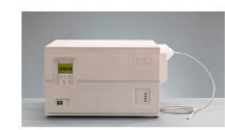

Gas Analysis

dynamic measurement of reaction gas streams catalysis and thermal analysis

molecular beam studies

, fermentation, environmental and ecological studies

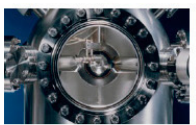

Surface Science UHVTPD

SIMS

end point detection in ion beam etch elemental imaging - surface mapoing

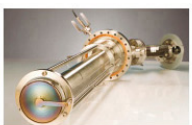

Plasma Diagnostics p plasma source characterization etch and deposition process reaction kinetic studies , analysis of neutral and radical species

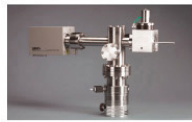

Vacuum Analysis - partial pressure measurement and control of process gases reactive sputter process control , vacuum diagnostics vacuum coating process monitoring 


\title{
Use of spectroscopic ellipsometry for feedback control during the growth of thin AlAs layers
}

\author{
M. Beaudoin, S. R. Johnson, M. D. Boonzaayer, and Y.-H. Zhang a) \\ Center for Solid State Electronics Research, Department of Electrical Engineering, \\ Arizona State University, Tempe, Arizona 85287-6206 \\ B. Johs \\ J. A. Woollam Co., Inc., 645 M Street, Lincoln, Nebraska 68508
}

(Received 5 October 1998; accepted 26 February 1999)

\begin{abstract}
Spectroscopic ellipsometry (SE) is an accurate in situ method for determining the composition and thickness of III-V semiconductor layers during growth. In order to achieve this control, an optical constant database is used to compare the modeled and experimental ellipsometric data. This procedure is very effective for controlling thickness and composition when the film has been growing for some time but is usually unreliable until several minutes into the growth (corresponding to $\sim 50 \mathrm{~nm}$ ). To use SE for the control of thinner layers $(1-20 \mathrm{~nm})$, a different approach has to be used. A new strategy is proposed which consists in looking at the raw SE signal in a limited wavelength range where the signal varies almost linearly with the film thickness. For AlAs grown on $\mathrm{GaAs}$, it is found the phase part of the SE signal for light between 2.5 and $2.8 \mathrm{eV}$ varies nearly linearly with the AlAs thickness. A series of AlAs/GaAs multiquantum well structures are grown and analyzed. The thickness determined by this use of the ellipsometric data are in close agreement with independent thickness measurements obtained from high resolution x-ray diffraction. (C) 1999 American Vacuum Society. [S0734-211X(99)06003-5]
\end{abstract}

\section{INTRODUCTION}

Many high speed electronic and optoelectronic devices rely on active regions whose thickness is on the order of monolayers. To integrate devices such as resonant tunneling diodes (RTD) ${ }^{1,2}$ into appropriate circuit architectures, a high run to run precision is required. ${ }^{3}$ In fact, for RTDs, even monolayer discrepancies in the barrier layer thicknesses can lead to unacceptably high variations in run to run device performance. ${ }^{3}$ In molecular beam epitaxy (MBE), the usual method used to control thin layers is with reflection high energy electron diffraction (RHEED) oscillations. Until very recently however, ${ }^{4,5}$ it was very difficult to obtain RHEED oscillations during substrate rotation. This prompted Zinck et $a l^{3,6}$ to use the photoemission oscillation (PEO) technique developed by Eckstein and co-workers ${ }^{7,8}$ because PEO can be easily acquired under substrate rotation. One drawback to both RHEED and PEO is that they can only be observed under layer by layer growth conditions and both are poorly suited to the control of thicker layers. A more universal feedback technique is therefore desirable.

Spectroscopic ellipsometry (SE) is an accurate method for measuring the thickness and composition of growing semiconductor films. $^{9-12}$ In MBE, SE has been successfully used in a feedback loop to grow Fabry-Perot cavities, ${ }^{10}$ latticematched InGaAs/InP ${ }^{11}$ and, recently, a complete resonant cavity enhanced photodetector (RCEPD). ${ }^{12}$ In SE, the signal is collected during growth and analyzed in real time using previously acquired optical constant database (OCDB) in order to extract the film's thickness and composition. This procedure is very effective when the film has been growing for

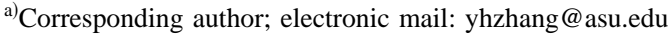

some time but is usually unreliable until several minutes into the growth (corresponding to approximately $50 \mathrm{~nm}$ ) and cannot, therefore, be used to control very thin layers such as quantum wells or the barrier layers of RTDs. It has, however, two main advantages over RHEED and PEO: (1) it is independent of the growth mode and (2) it can be used for control of thick devices. To extend the use of SE to the control of much thinner layers, an approach different from the one based on comparison with an OCDB needs to be developed.

In this article we propose to consider the raw SE data in a limited wavelength range where the SE parameters vary close to linearly with the film thickness. A series of GaAs/ AlAs multiquantum well structures (MQW) were grown in order to independently assess the layer thicknesses by $\mathrm{x}$-ray diffraction (XRD) rocking curves. A sample consisting of $11.7 \mathrm{~nm}$ AlAs barriers was used to calibrate the technique. An algorithm is then developed and used to measure the thickness of two other samples with different AlAs barrier thicknesses. These thicknesses are in excellent agreement with those directly determined from independent XRD measurements.

\section{EXPERIMENT}

The experiments were carried out on a $\mathrm{DCA}^{\mathrm{TM}}$ molecular beam epitaxy (MBE) chamber equipped with ellipsometer ports as has been described previously. ${ }^{10-12}$ The SE signal is given by the ratio of the complex reflectivities $r_{p}$ and $r_{s}$ where the subscripts $p$ and $s$ refer to light polarized parallel and perpendicular to the plane of incidence, respectively,

$$
\rho=\frac{r_{p}}{r_{s}}=\frac{\left|r_{p}\right|}{\left|r_{s}\right|} e^{i\left(\delta_{p}-\delta_{s}\right)}=\tan (\Psi) e^{i \Delta} .
$$


TABLE I. AlAs thickness measurement using the variation of $\Delta$ parameter in the range between 2.5 and $2.8 \mathrm{eV}$. The last column gives the average thickness and standard deviations for the 13 wavelengths in the range of 2.5-2.8 $\mathrm{eV}$. This column clearly shows that the algorithm is able to determine thickness precisely.

\begin{tabular}{cccc}
\hline \hline $\begin{array}{c}\text { HRXRD } \\
\text { thickness }(\mathrm{nm})\end{array}$ & $\begin{array}{c}\text { Growth time } \\
(\mathrm{s})\end{array}$ & $\begin{array}{c}\text { Time base } \\
\text { thickness }(\mathrm{nm})\end{array}$ & $\begin{array}{c}\text { Thickness from } \\
\text { SE } \Delta(\mathrm{nm})\end{array}$ \\
\hline $11.7 \pm 0.2$ & 200 & $\ldots$ & $\ldots$ \\
$5.5 \pm 0.3$ & 100 & 5.9 & $5.49 \pm 0.08$ \\
$3.0 \pm 0.4$ & 50 & 2.9 & $2.56 \pm 0.05$ \\
\hline \hline
\end{tabular}

The SE signal is most conveniently expressed mathematically by an amplitude, $\tan (\Psi)$, and phase, $\Delta$, difference between the $p$ and $s$ components of the electric field. The $\Psi$ and $\Delta \mathrm{SE}$ parameters are sensitive to the structure (thickness, composition, etc.) and the optical constants of the growing film. ${ }^{13}$ The signal is acquired with a J. A. Woollam M88 ellipsometer in the rotating analyzer configuration (88 wavelengths ranging from 277 to $765 \mathrm{~nm}$ ) using their WVASETM control and acquisition software.

A series of three GaAs/AlAs samples were grown by solid source MBE using an $\mathrm{As}_{4}$ overpressure. The samples were grown on semi-insulating GaAs and consisted of a GaAs buffer layer, a $100 \mathrm{~nm}$ AlAs layer, and an $100 \mathrm{~nm}$ GaAs layer followed by a 10 period GaAs/AlAs multiple quantum well (MQW) structure. Each well in the MQW was nominally kept at $33.0 \mathrm{~nm}$ while the AlAs barriers were grown with different growth times as reported in Table I. The growths were carried out at a substrate temperature of $600^{\circ} \mathrm{C}$, as controlled by optical band edge thermometry..$^{4,15}$ The $100 \mathrm{~nm}$ AlAs and GaAs layers were grown under conventional OCDB based SE feedback control. ${ }^{10-12}$ The MQW structures were grown under time-based control and SE was used to monitor the growth. The sample structures were assessed by double-crystal x-ray diffraction rocking curves and analyzed by simulations using dynamical diffraction theory. ${ }^{16}$

\section{RESULTS AND DISCUSSION}

Figure 1 shows the time variation of the $\Psi$ parameter for three wavelengths of light (expressed in $\mathrm{eV}$ ) during the growth of a sample with $11.7 \mathrm{~nm}$ AlAs barrier layers. The sample cross-section is shown in the inset of Fig. 2. The last GaAs cap layer, which should show a maximum in the $\Psi$ signal, is not seen as the WVASE buffer filled up and data collection stopped. The $2.6 \mathrm{eV} \Psi$ curve shows a nearly linear down sloping segment during the growth of each AlAs barrier. The first AlAs barrier, grown on top of the $100 \mathrm{~nm}$ GaAs layer begins at a lower value than the remaining nine barriers grown on top of a thinner GaAs quantum well. Its slope does appear to be very close to that of the last nine barriers however. Figure 1 also shows the different periods of the interference fringes caused by the different optical paths for light of different wavelengths. During the growth of the MQW structure, the $\Psi$ signal is very periodic at the shorter wavelengths while the longer wavelength signal

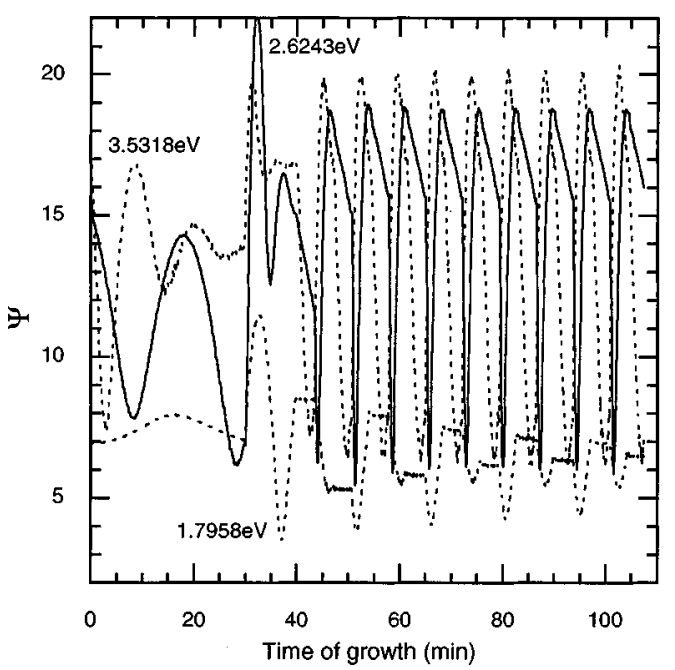

FIG. 1. Variation of the amplitude parameter, $Y$, of the SE signal with time during the growth of a GaAs/AlAs MQW structure. The SE data is first acquired during the growth of a $100 \mathrm{~nm}$ AlAs layer and a $100 \mathrm{~nm} \mathrm{GaAs}$ layer before the MQW growth.

shows an additional modulation. The additional modulation comes from the fact that longer wavelength light penetrates deeper in the film and "sees" more periods whereas the shorter wavelengths are more sensitive to the film thickness near the surface. In fact, for the shortest wavelength light shown in Fig. $1(3.5 \mathrm{eV})$, the optical path is such that an interference fringe is visible in the AlAs layer. Since the signal at $2.6 \mathrm{eV}$ appears nearly linear during the growth of the AlAs layer, a simple thickness determination algorithm may be developed.

Figure 2 shows the XRD rocking curve for the sample structure shown in the inset. Also shown, shifted down for clarity, is the result of a fit based on dynamical diffraction theory. ${ }^{16}$ The agreement between the experimental and simulated patterns are excellent. The MQW structure satellite peaks and the shorter period thickness interference fringes

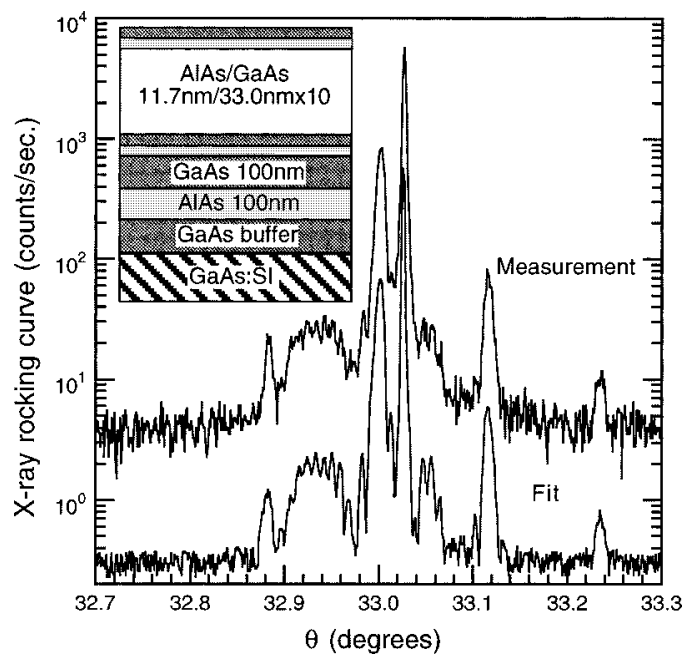

FIG. 2. X-ray rocking curve of a GaAs/AlAs MQW structure. The inset shows the sample structure. The bottom curve is the simulated pattern from dynamical diffraction theory and is shifted down for clarity. 
due to the $100 \mathrm{~nm} \mathrm{AlAs}$ and GaAs layers are clearly visible in the experimental pattern and exactly reproduced by the simulation. Extracting the thickness of the AlAs and GaAs layers of the MQW structure is simplified by the fact that only binaries are used in each layer. The splitting between the zeroth order satellite peak and the main GaAs Bragg peak, which gives the average MQW lattice constant, can thus be used directly to compute the AlAs/GaAs thickness ratios. This was done using 0.56535 and $0.56618 \mathrm{~nm}$ for the lattice constants of GaAs and AlAs, respectively; these are widely used values. This analysis has been done on the three samples presented here. In order to use the thickness algorithm meaningfully, the GaAs quantum well thicknesses had to be kept constant for the entire series. The uncertainty in the total AlAs + GaAs thickness for each period is $0.1 \mathrm{~nm}$ as determined visually from the fit using dynamical diffraction theory. For very thin AlAs layers, the superlattice zeroth order sattelite peak nearly coincides with the substrate peak; dynamical theory fits with different thickness ratios were qualitatively compared with the experimental patterns to determine the uncertainties in AlAs thickness. The XRD results with their associated uncertainties are reported in Table I.

Figures 3(a) and 3(b) show blowups of the time variations of the $\Delta$ and $\Psi$ SE parameters for the same three wavelengths shown in Fig. 1. The blowups cover the growth of three AlAs barriers as shown on the figures by the doubleended arrows. In order to develop an algorithm for thickness determination, we chose to concentrate on wavelength ranges where the SE signal showed a nearly linear variation. Figure 3 show even more clearly that long wavelength light displays interference fringes which span several alternating layers (i.e., long wavelength light sees deeper below the surface). On the other hand, the short wavelength light, although periodic with the layer thicknesses, shows elaborate interference oscillation shapes. The $2.6 \mathrm{eV}$ light shows a nearly linear dependence on time (thickness) with a more pronounced variation in its $\Delta$ parameter than in its $\Psi$ parameter; hence, the $\Delta$ variation is more sensitive to thickness variation than the corresponding $\Psi$ variation. With further analysis, we determined that light between 2.5 and $2.8 \mathrm{eV}$ showed a nearly linear response with film thickness and was easy to analyze. One should note "glitches" on either side of the AlAs layers where both the $\Delta$ and $\Psi$ parameters are constant in time. These glitches were intentionally introduced with growth interrupts in order to highlight the AlAs layers and simplify the analysis.

Figure 4 shows the variation of $\Delta$ with AlAs thickness for the $11.7 \mathrm{~nm}$ AlAs barriers sample for light between 2.5 and $2.8 \mathrm{eV}$. This range represents 13 of the 88 wavelengths available with the M88 ellipsometer. The total AlAs thickness is determined from XRD and a constant growth rate is assumed throughout the AlAs layer growth; the thickness axis is therefore a simple translation of time to thickness and its magnitude is only meant to be valid over the AlAs interval. Assuming a constant growth rate neglects the effect of shutter transients which we expect to be small in this case anyway. Figure 4 clearly shows the effect of the growth inter-
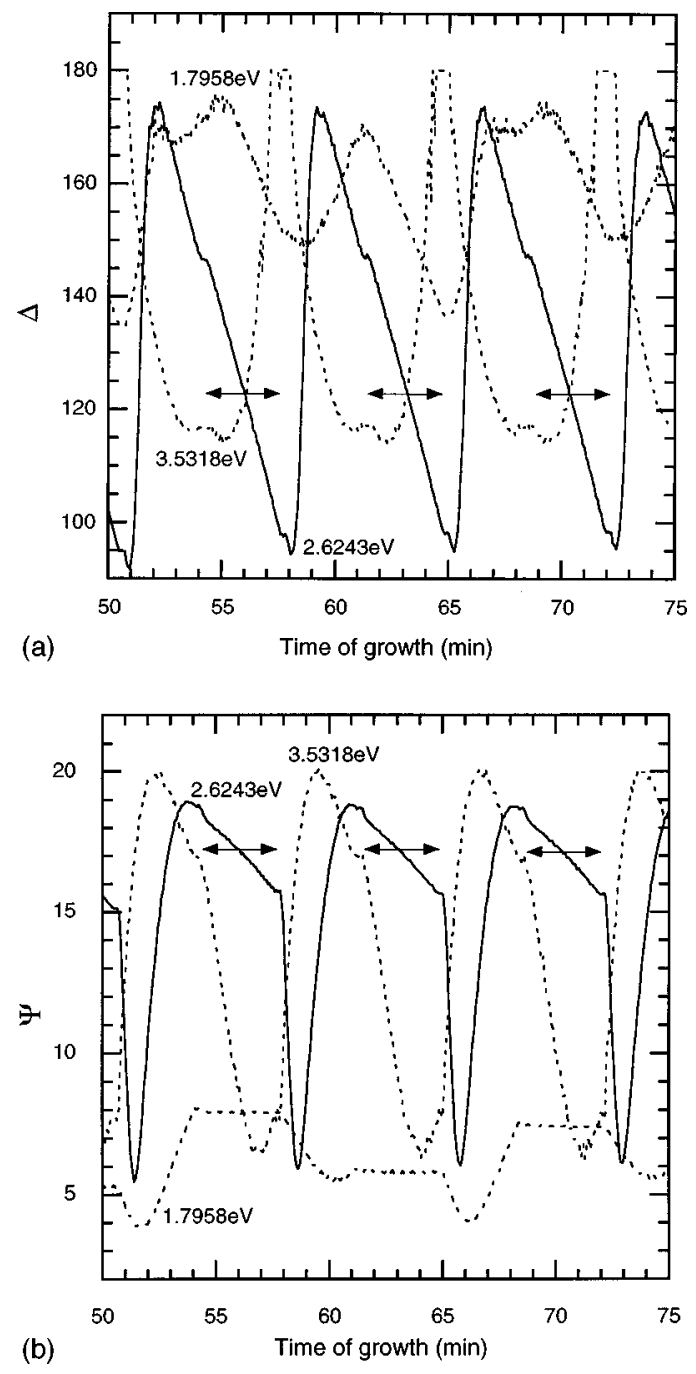

FIG. 3. Time variation of the SE parameters (a) $\Delta$ and (b) $\Psi$ over a few AlAs layers for three wavelengths of light. The variation of both $\Delta$ and $\Psi$ are nearly linear for light of $2.6 \mathrm{eV}$.

rupts and the nearly linear variation of $\Delta$ with thickness during the AlAs growth. The $\Delta$ variation was modeled with a quadratic, as a function of thickness $x$, for each of the 13 wavelengths included in the interval:

$$
\Delta=c x^{2}+b x+\Delta_{0} .
$$

One has to be careful when extending the results of the quadratic fits to determine the thickness of other samples. In fact, this numerical algorithm is only expected to be valid for AlAs layers grown on GaAs layers of the same thickness as those grown here! In general, the quadratic relationships are expected to depend on the initial $\Delta_{0}$ value. As the first AlAs layer in Fig. 1 shows, this variation appears almost independent of its starting value but more work will be required to clarify this point and determine if a universal algorithm is possible or if a different algorithm will need to be developed for each different structure that one wishes to grow. Even in the case of our nominally identical structures, we found a $1 \%$ variation in the initial $\Delta_{0}$ values for MQW samples grown under time base control. 


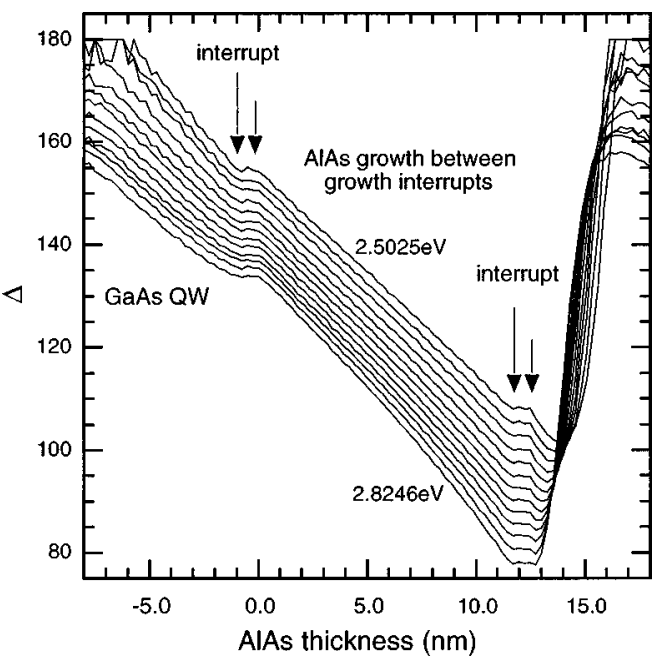

FIG. 4. Variation of $\Delta$ at several wavelengths with AlAs layer thickness for one period of a MQW sample with $11.7 \mathrm{~nm}$ AlAs barriers sandwiched between GaAs layers. Vertical arrows show the growth interruptions at both interfaces. At all wavelengths, $\Delta$ varies nearly linearly with thickness although a quadratic correction improves the fit (not shown).

Equation (2) shows that $\Delta$ will vary in a prescribed way with the AlAs film thickness. To subtract out the $1 \%$ variations in $\Delta_{0}$, we invert Eq. (2) using $\delta \Delta=\Delta-\Delta_{0}$ :

$$
x=\left(\sqrt{\frac{b^{2}}{4 c^{2}}-\frac{\delta \Delta}{c}}\right)-\frac{b}{2 c},
$$

where $x$ is the AlAs thickness while $b$ and $c$ are fixed from the fit to the calibration sample (11.7 nm AlAs barriers). Equation (3) can now be applied to the other two samples. The results are reported in Table I where they are also compared to the XRD results. As Table I shows, the agreement is excellent. The last column of Table I shows that the standard deviation in the 13 wavelengths average is smaller than the uncertainty of the independent XRD thickness measurements. In this case, the advantage of using SE instead of time base control is not clear. What is clear however is that the idea of using the variation of $\Delta$ does work. In the next series of planned experiments, the $\mathrm{Al}$ cell temperature will be varied between samples so that time base control becomes more difficult and the sample structures will be designed to minimize the uncertainty in XRD measurements. These experiments will constitute a more stringent test of the proposed idea. Samples with varying GaAs quantum well thicknesses will also be used in order to verify the sensitivity of Eq. (3) to variations in $\Delta_{0}$.

\section{CONCLUSION}

An algorithm has been proposed which promises to extend the applicability of spectroscopic ellipsometry to thickness measurements in layers as thin as $3 \mathrm{~nm}$, such as those found in quantum wells or barriers. The algorithm uses the initial variation of the raw ellipsometric signal in a range of wavelengths where the signal is nearly linear. For AlAs barriers grown on GaAs, the proposed algorithm gave thickness results in excellent agreement with independent assessments by $\mathrm{x}$-ray diffraction. More investigation is required in order to determine if the proposed algorithm is universal or if it is material and structure specific.

\section{ACKNOWLEDGMENTS}

This work was supported by DARPA under Contract No. MDA 972-95-1-0016 managed by Lt. Col. Gernot S. Pomrenke.

${ }^{1}$ E. R. Brown, J. R. Söderström, C. D. Parker, L. J. Mahoney, K. M. Molvar, and T. C. McGill, Appl. Phys. Lett. 58, 2291 (1991).

${ }^{2}$ E. Özbay, D. M. Bloom, D. H. Chow, and J. N. Schulman, IEEE Electron Device Lett. 14, 400 (1993).

${ }^{3}$ J. J. Zinck, D. H. Chow, J. N. Schulman, and H. L. Dunlap, Appl. Phys. Lett. 68, 1406 (1996).

${ }^{4}$ W. Braun, H. Möller, and Y.-H. Zhang, J. Vac. Sci. Technol. B 16, 1507 (1998).

${ }^{5}$ W. Braun, H. Möller, and Y.-H. Zhang, Appl. Phys. Lett. 74, 138 (1999).

${ }^{6}$ J. J. Zinck and D. H. Chow, J. Cryst. Growth 175/176, 323 (1997).

${ }^{7}$ J. N. Eckstein, C. Webb, S.-L. Weng, and K. A. Bertness, Appl. Phys. Lett. 51, 1833 (1987).

${ }^{8}$ J. N. Eckstein, C. Webb, S.-L. Weng, and K. A. Bertness, J. Vac. Sci. Technol. B 6, 736 (1988).

${ }^{9}$ D. E. Aspnes, Surf. Sci. 307-309, 1017 (1994).

${ }^{10}$ C.-H. Kuo, M. D. Boonzaayer, M. F. DeHerrera, D. K. Schroder, G. N. Maracas, and B. Johs, J. Cryst. Growth 175/176, 281 (1997).

${ }^{11}$ C.-H. Kuo, M. D. Boonzaayer, M. DeHerrera, T. Kyong, Y.-H. Zhang, B. Johs, and J. S. Hale, J. Vac. Sci. Technol. B 16, 1484 (1998).

${ }^{12}$ M. Beaudoin, P. Kelkar, M. D. Boonzaayer, W. Braun, P. Dowd, S. R. Johnson, U. Koelle, C.-M. Ryu, and Y.-H. Zhang, J. Cryst. Growth (in press).

${ }^{13}$ G. N. Maracas, C.-H. Kuo, S. Anand, R. Droopad, G. R. L. Sohie, and T. Levola, J. Vac. Sci. Technol. A 13, 727 (1995).

${ }^{14}$ M. K. Weilmeier, K. M. Colbow, T. Tiedje, T. VanBuuren, and L. Xu, Can. J. Phys. 69, 422 (1991).

${ }^{15}$ S. R. Johnson, C. Lavoie, M. K. Niessen, and T. Tiedje, US Patent No. 5,388,909 (1995).

${ }^{16}$ P. F. Fewster, Philips J. Res. 45, 620 (1984). 